\title{
Mayer-Rokitansky-Kuster-Hauser syndrome associated with serous papillary cystadenocarcinoma of the ovary
}

\author{
G R C Silva ${ }^{1}$, W U S Wickramasinghe ${ }^{1}$, V P Gange ${ }^{1}$ \\ Sri Lanka Journal of Obstetrics and Gynaecology 2010; 32: 91-92
}

\section{Introduction}

The Mayer-Rokitansky-Kuster-Hauser (MRKH) syndrome occurs in 1 out of 4500 female births. It is characterized by congenital aplasia of the uterus and the upper part $(2 / 3)$ of the vagina in women showing normal development of secondary sexual characteristics with a normal 46XX karyotype. Their ovaries and fallopian tubes are normal. The coelomic epithelium which creates the ovaries develops independently of the Mullerian duct which creates the uterus, cervix and 2/3 of the upper vagina. Therefore, individuals with MRKH syndrome have normal ovaries and fallopian tubes and they are at a normal risk of developing ovarian malignancy.

\section{Case report}

A 47-year old unmarried woman was admitted to the gynaecological casualty unit at de Zoysa Maternity Hospital in November 2010 with complaints of abdominal pain for 2 weeks. She stated that she has never had menstrual periods. As she has not sought medical help, she was not investigated for amenorrhoea before. She had neither fever nor associated bowel or urinary symptoms. There was no family history of genital tract malignancies. On examination she was $150 \mathrm{~cm}$ tall and weighing $55 \mathrm{~kg}$. Her cardiovascular system was normal. Gynaecologic examination revealed complete vaginal agenesis. Phenotypic sex was female; pubic hair and breast development were Tanner stage 5. Abdominal and rectal examination revealed a cystic non tender, mobile mass extending from pelvis corresponding to about 20 weeks size gravid uterus. Serum CA-125 level was elevated $(2930 \mathrm{U} / \mathrm{ml})$. Ultrasound scan showed $15 \times 15 \times 12 \mathrm{~cm}$ cystic mass with solid areas and multiple septae. Uterus could not be identified. There was no ascitic fluid. Both kidneys were normal in echogenicity. Intravenous urographic examination (IVU) showed a normal urinary tract. At laparatomy, partly cystic well-shaped mass was found arising from left adenaxae. Uterus was formed of bilaterally rudimentary uterine bulbs. Left side was larger than

\footnotetext{
${ }^{1}$ De Zoysa Hospital for Women

Correspondence: Prasantha Gange

E-mail: gangep@hotmail.com
}

right. Right fallopian tube and right ovary appeared normal. Left fallopian tube was swollen and adhered to ovarian mass arising from left ovary. Tumor and the rudimentary uterine bulb was excised and sent for histology. Ascitic fluid was sent for cytology. There were no enlarged lymph nodes or tumor deposits in the abdomen or in the pelvis. Histopathological evaluation of the specimen revealed a rudimentary uterus with moderately differentiated serous papillary cystadenocarcinoma of the ovary. Further surgery is planned for a pelvic clearance prior to chemotherapy. Treatment MRHK syndrome depends on the symptoms, associated anomalies and the age of the patient. If the patient suffers pelvic pain due to a rudimentary horn, resection by laparotomy or laparoscopy may resolve the problem. If the diagnosis is made on an amenorrheic young girl, vaginoplasty could be done.

\section{Discussion}

Common presentations of MRKH syndrome are primary amenorrhoea, sterility and pelvic pain. Developing an ovarian neoplasm together with the syndrome is an unexpected rare state. For a long time the syndrome has been considered as s sporadic anomaly $^{1}$, but increasing number of familial cases now support the hypothesis of a genetic cause ${ }^{2}$. But the etiology of MRKH syndrome still remains unclear. MRKH syndrome is subdivided into two types: type I (isolated) or Rokistasky sequence, and type II or MURCS association (Mullerian duct aplasia, renal dysplasia and cervical somite anomalies). MURCS association is more frequently associated with renal, vertebral, and, to a lesser extent, auditory, cardiac defects and digital anomalies (syndactyly, polydactyly). Type I MRKH syndrome is less frequent than MURCS association ${ }^{3}$. Complete aplasia of the uterus in the presence of two rudimentary horns linked by a peritoneal fold and normal fallopian tubes correspond to isolated MRKH syndrome type I. Type II MRKH syndrome is characterized by symmetric or asymmetric uterine hypoplasia, accompanied by aplasia of one of the two horns or by a size difference between the two horn rudiments, coupled with tubular malformations such as hypoplasia or aplasia of one or both tubes. Cases of polycystic ovaries ${ }^{4}$ and ovarian tumors ${ }^{5}$ have been described in women presenting with MRKH syndrome type II. 


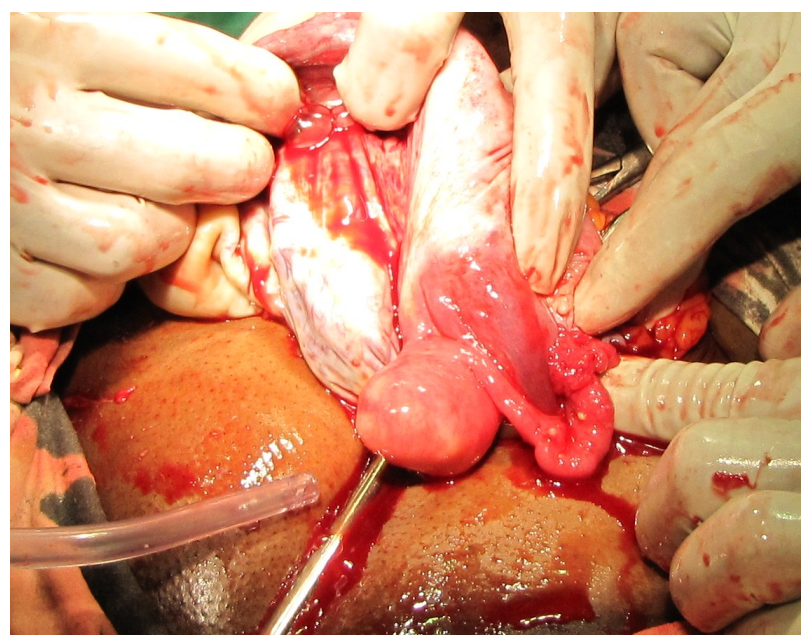

Figure 1. Cyst and rudimentary horn.

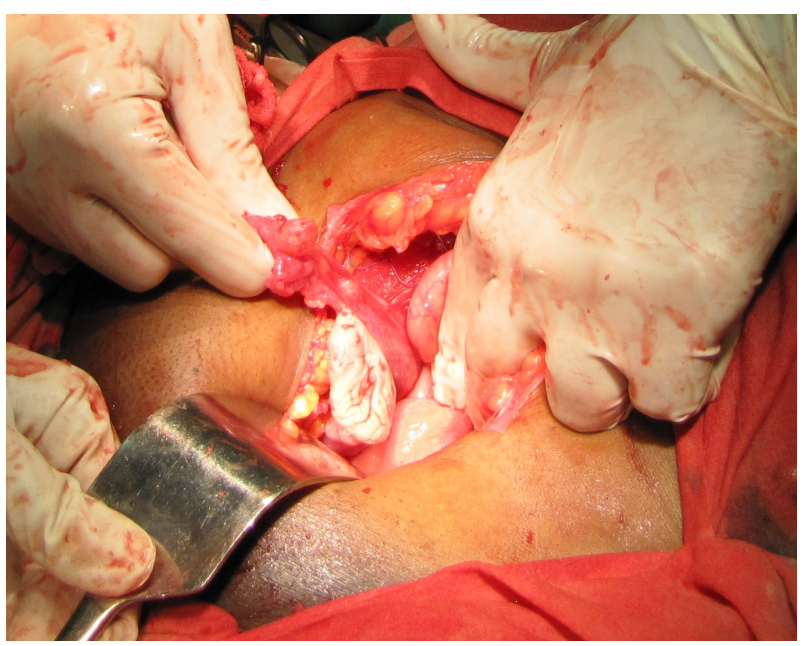

Figure 2. Other ovary, tube and rudimentary horn.

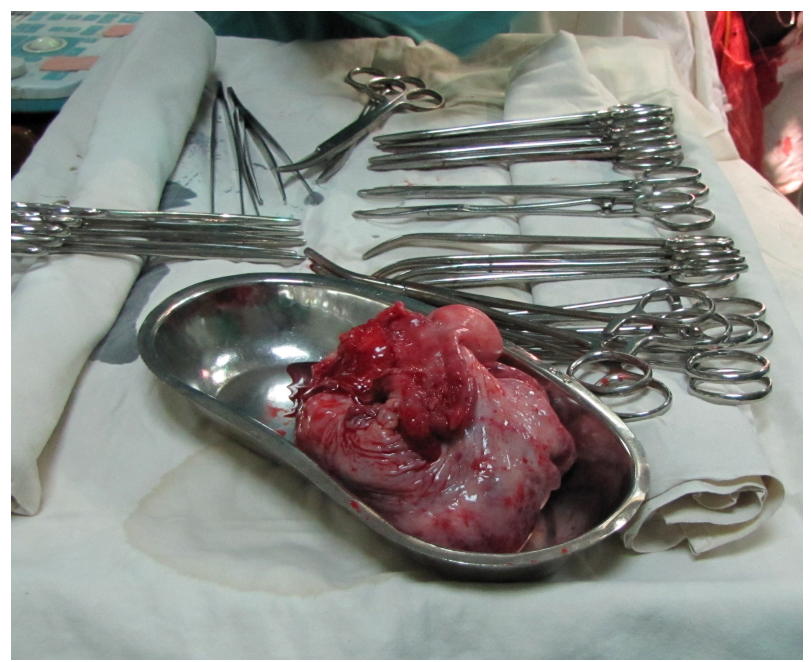

Figure 3. Removed specimen.

\section{Conclusion}

Ovarian neoplasms can arise in patients with MRKH syndrome although this is rare. Their risk of developing gynaecological malignancies is no different from that of normal female population ${ }^{6}$.

\section{Referrences}

1. Carson SA, Simpson JL, Malinak LR, Elias S, Gerbie AB, Buttram VC Jr, Sarto GE. Heritable aspects of uterine anomalies. II. Genetic analysis of Mullerian aplasia. Fertil Steril 1983; 40: 86-90.

2. Jones HW, Mermut S. Familial occurrence of congenital absence of the vagina. Am J Obstet Gynecol 1972; 114: 1100-01.
3. Strubbe EH, Cremers CW, Willemsen WN, Rolland R, Thijn CJ. The Mayer-Rokitansky-Kuster-Hauser (MRKH) syndrome without and with associated features: two separate entities? Clin Dysmorphol 1994; 3: 192-9.

4. Ugur M, Karakaya S, Zorlu G, ArslanS, Gulerman C, Kukner $\mathrm{S}$, Gokmen O. Polycystic ovaries in association with mullerian anomalies. Eur J Obstet Gynecol Reprod Biol 1995; 62: $57-9$.

5. Ghirardini G, Magnani A. Mayer-Rokitansky-Kuster-Hauser syndrome and ovarian cancer. Report of a case. Clin Exp Obstet Gynecol 1995; 22: 247-8.

6. Koonings PP, Marayati L, Schlaerth JB, Lobo RA. MayerRokitansky-Kuster-Hauser syndrome associated with endodermal sinus tumor of the ovary. Fertility Sterility 1991 Sep; 56(3): 577-8. 